\title{
A Comparative Study of Tensile Strength of Three Operative Fixation Techniques for Metacarpal Shaft Fractures in Adults: A Cadaver Study
}

\author{
Jin Rok Oh, MD, Doo Sup Kim, MD, Jun Seop Yeom, MD, Sang Kyu Kang, MD, Yun Tae Kim, MD* \\ Department of Orthopedic Surgery, Wonju Severance Christian Hospital, Yonsei University Wonju College of Medicine, Wonju, \\ ${ }^{*}$ Center of Biomedical Data Science, Yonsei University Wonju College of Medicine, Wonju, Korea
}

Background: We sought to estimate the ultimate tensile strength after metacarpal shaft fracture repair in adults using three operative fixation methods: plate fixation, Kirschner wire (K-wire) fixation, and intramedullary headless compression screw fixation. We also compared the advantages and disadvantages of each operative technique.

Methods: We acquired 30 metacarpal bones from four Korean adult cadavers without trauma, operative history, or deformities. The 30 metacarpal bones were divided into ten groups consisting of three metacarpal bones each with matching sizes and lengths. They were fractured, reduced, and fixed with plate and screws, K-wires, or headless compression screws. We performed force testing, collected ultimate tensile strength data, and created a stress-strain graph.

Results: The ultimate tensile strength of ten groups according to the fixation method was as follows: late and screw fixation, $246.1 \mathrm{~N}$ (range, 175.3 to $452.4 \mathrm{~N}$ ); K-wire fixation, $134.6 \mathrm{~N}$ (62.7 to $175.0 \mathrm{~N}$ ); and intramedullary headless compression screw fixation, 181.2 N (119.2 to $211.7 \mathrm{~N}$ ). The median tensile strength of each fixation method was significantly different. In addition, the post-hoc test showed significant difference between the plate and screw fixation and K-wire fixation, between the headless compression screw fixation and K-wire fixation, and between the headless compression screw fixation and plate and screw fixation. Conclusions: The tensile strength median values decreased in the following order showing significant differences among the fixation methods: plate and screw fixation, headless compression screw fixation, K-wire fixation. Significant differences were also observed between the plate and screw fixation and K-wire fixation, between the headless compression screw fixation and K-wire fixation, and between the headless compression screw fixation and plate and screw fixation.

Keywords: Metacarpal, Metacarpal shaft fracture, Cadaver, Headless compression screw

Metacarpal shaft fractures are a common hand injury that is mostly self-contained. Therefore, conservative treatments such as closed reduction and splinting are effective for metacarpal shaft fractures without misalignment. ${ }^{1,2)}$ These treatments are effective because metacarpal bones

Received July 11, 2018; Accepted September 17, 2018

Correspondence to: Doo Sup Kim, MD

Department of Orthopedic Surgery, Wonju Severance Christian Hospital, Yonsei University Wonju College of Medicine, 20 IIsan-ro, Wonju 26426, Korea

Tel: +82-33-741-0114, Fax: +82-33-736-7326

E-mail: dskim1974@yonsei.ac.kr are highly stable at their attachment points to many intrinsic muscles and ligaments. Furthermore, sufficient blood supply requires shorter healing time and decreases the chance of nonunion of the fracture. ${ }^{3)}$ However, complications can arise from long-term joint fixation after synostosis such as ankylosis.

In contrast, there is a high probability of angular and rotational deformities in the case of reduction and casting of unstable fractures. ${ }^{4,5}$ If these deformities are not surgically corrected, serious aesthetic and functional problems such as cross-fingering and superimposition may occur. ${ }^{6}$ By adapting the Kirschner wire (K-wire) technique, different approaches can be used depending on the technique, 
plate used, internal and external fixation methods, fracture position, fracture condition, soft tissue condition, and most importantly, the surgeon's preference. Among various methods, plate fixation has shown the most promising results. ${ }^{7)}$ However, large incisions can lead to problems such as soft tissue injury, ${ }^{8,9)}$ extension delay, tendon irritation, and tendon rupture. ${ }^{6,710-12)}$ By contrast, percutaneous K-wire fixation causes less soft tissue damage and has lower infection rates, but fixation strength is comparatively weak; therefore, additional splinting may be required, subsequently causing soft tissue restraint. ${ }^{4,6}$

Intramedullary headless cannulated compression screws for metacarpal shaft fractures were designed to complement the weaknesses of the plate fixation and $\mathrm{K}$ wire fixation methods. In this study, metacarpal shaft fractures were induced on cadavers, and the headless compression screw fixation, plate fixation, and K-wire fixation methods were applied to assess and compare the tensile strength and effectiveness of the headless cannulated compression screw fixation method with other methods.

\section{METHODS}

We used four Korean adult cadavers with no history of trauma, surgeries, or deformities on their hands. Excluding the first finger, we chose and sorted 30 other metacarpal bones into ten groups, each of which was composed of three metacarpal bones according to similar length and thickness. Three metacarpal bones of each group were induced transverse type shaft fractures with a saw. After reduction, plate fixation, K-wire fixation, and headless cannulated compression screw fixation were performed. We used an I plate $(1.0 \mathrm{~mm}$ in thickness; Jeil Medical, Seoul, Korea), self-tapping cortical screws $(2.0 \mathrm{~mm}$ in diameter; Jeil Medical), an Arix headless cannulated compression screw (3.0 mm in diameter, Jeil Medical), and a 1.6- $\mathrm{mm} \mathrm{K-}$ wire (1.6 mm in thickness; Depuy Synthes, Raynham, MA, USA) (Fig. 1). As the I plate had 16 holes, we cut that into an appropriate length to have 8 to 9 holes. With the prepared plate in place, cortical screws were inserted from the dorsal side to the volar side. Intramedullary K-wire fixation was performed from the metacarpal base side. The intramedullary headless compression screw $(3.0 \mathrm{~mm}$ in diameter, Jeil medical) was inserted from the metacarpal head to the base, the thread of which crossed the fracture line (Fig. 2). Each fixation method for the metacarpal shaft fracture was assessed using a force test system, Multitest 2.5-i (ILC 1000N [Loadcell]; Mecmesin, Horsham, UK) (Fig. 3). Both ends of the metacarpal bone were firmly fixed to reduce the rotation force when measuring the tensile strength. Stress was applied to fixed sites until displacement was observed, and the amount of applied stress
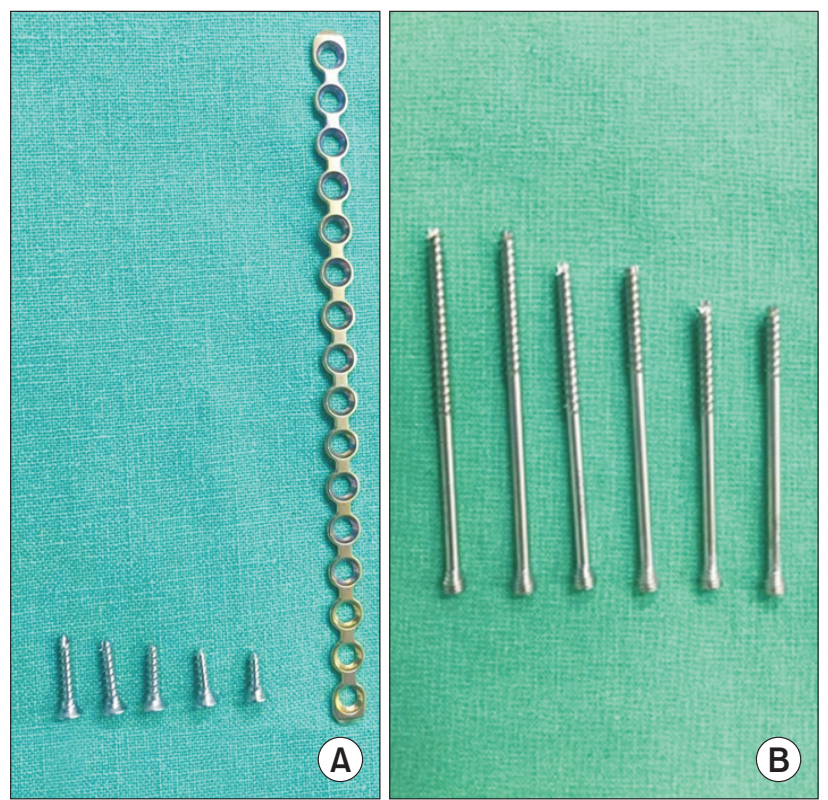

Fig. 1. (A) I plate (1.0 mm in thickness; Jeil Medical, Seoul, Korea) and self-tapping cortical screws (2.0 mm in diameter, Jeil Medical). (B) Arix headless cannulated compression screws $(3.0 \mathrm{~mm}$ in diameter, Jeil Medical).
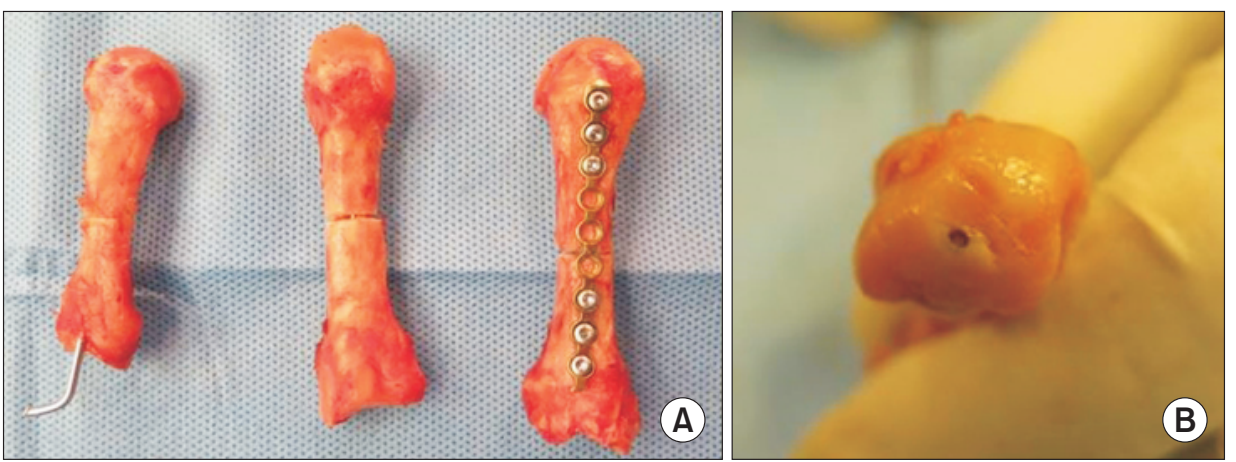

Fig. 2. (A) Three metacarpal shaft fractures of the cadaver bones fixed with the Kirschner wire (K-wire), headless compression screw, or plate fixation (from left to right). (B) Articular surface of the metacarpal head fixed with the headless compression screw method. This screw does not protrude and has minimal invasion of the articular surface area. 
Oh et al. Tensile Strength of Three Fixation Methods in Metacarpal Fractures

Clinics in Orthopedic Surgery • Vol. 11, No. 1, 2019•www.ecios.org

and displacement values were graphed (Figs. 4 and 5).

\section{RESULTS}

The average values for the ultimate tensile strength measured from the ten groups were as follows: plate and screw fixation, $246.1 \mathrm{~N}$ (range, 175.3 to $452.4 \mathrm{~N}$ ); K-wire fixation, $134.6 \mathrm{~N}$ (range, 62.7 to $175.0 \mathrm{~N}$ ); and intramedullary headless compression screw fixation, $181.2 \mathrm{~N}$ (range, 119.2 to $211.7 \mathrm{~N}$ ) (Tables 1 and 2). The ultimate tensile strength for three fixation methods were compared: there were significant differences in the median values $(p<0.05)$ (Table 2). In order to know whether there is significant difference between two fixation methods, we performed

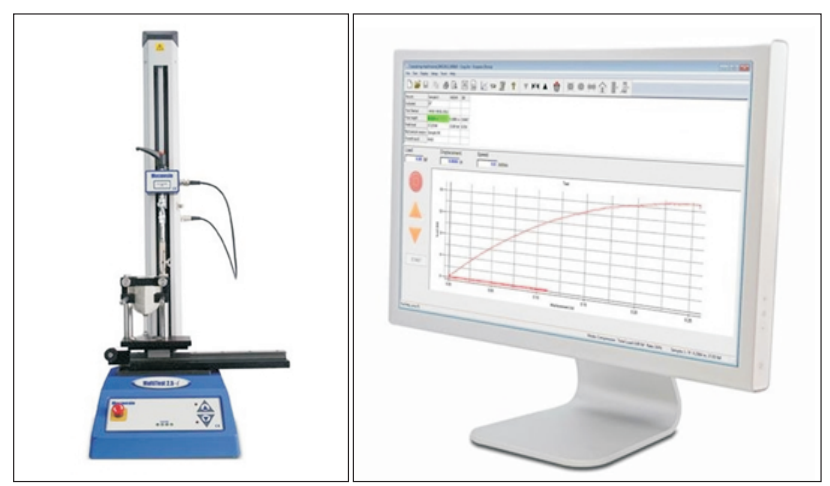

Fig. 3. Force test system (Multitest 2.5-i; Mecmesin, Horsham, UK). post-hoc test: significant differences were found between the plate and screw fixation and K-wire fixation, between the plate and screw fixation and headless compression screw fixation, and between $\mathrm{K}$-wire fixation and headless compression screw fixation (Table 3). Considering the small sample size, instead of finding a normal distribution, the medians and ranges (minimum to maximum) were calculated, and the Kruskal-Wallis test, a nonparametric

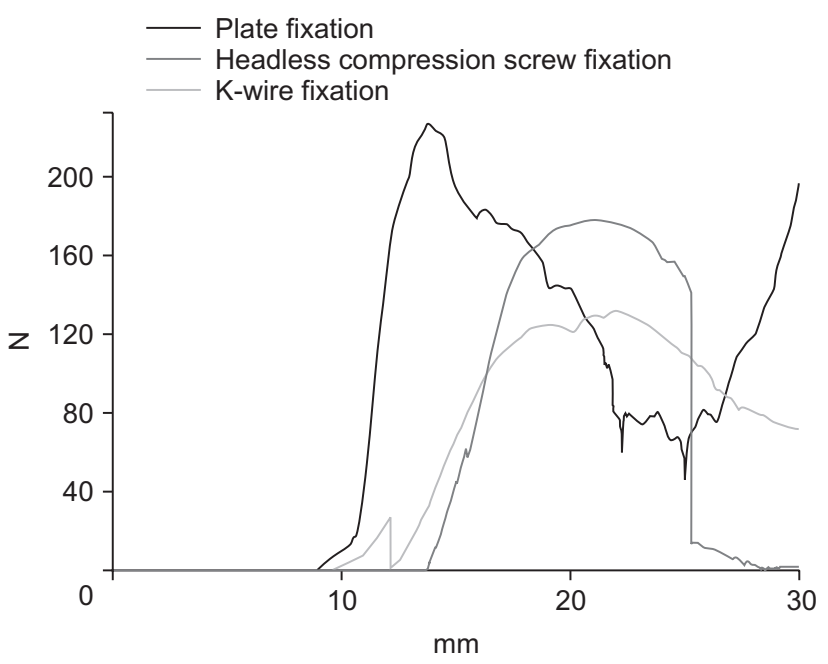

Fig. 5. Stress-strain graph based on force testing of three metacarpal bone fracture sites in group 2, each of which was fixed with a plate, Kirschner wire (K-wire), or headless compression screw.
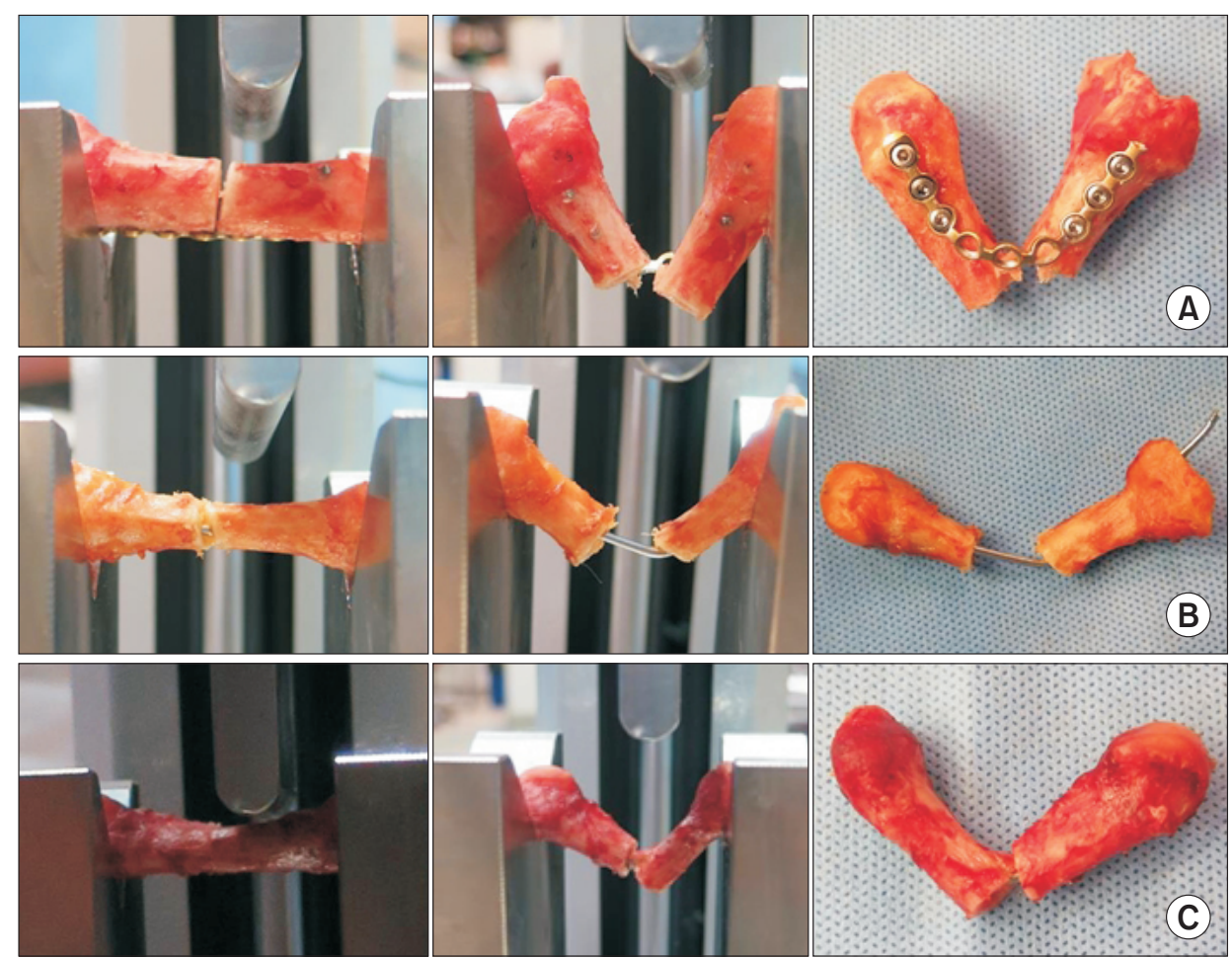

Fig. 4. Measurement of tensile strength by the force test system. (A) Plate fixation. (B) K-wire fixation. (C) Headless compression screw fixation. 
Oh et al. Tensile Strength of Three Fixation Methods in Metacarpal Fractures

Clinics in Orthopedic Surgery • Vol. 11, No. 1, 2019•www.ecios.org

\begin{tabular}{cccc|}
$\begin{array}{c}\text { Table 1. Ultimate Tensile Strength in Force Testing of Ten Groups } \\
\text { of Fractures Fixed with Plate Fixation, K-Wire Fixation, } \\
\text { and Headless Compression Screw Fixation }\end{array}$ \\
\hline \multirow{4}{*}{ Group } & \multicolumn{3}{c}{ Ultimate tensile strength (N) } \\
\cline { 2 - 4 } & $\begin{array}{r}\text { Plate and } \\
\text { screw }\end{array}$ & K-wire & $\begin{array}{c}\text { Headless } \\
\text { compression screw }\end{array}$ \\
\hline 1 & 452.4 & 158.5 & 195.0 \\
\hline 2 & 227.2 & 131.8 & 178.5 \\
\hline 3 & 176.7 & 144.5 & 211.7 \\
\hline 4 & 254.1 & 131.4 & 184.0 \\
\hline 5 & 272.3 & 175.0 & 119.2 \\
\hline 6 & 175.3 & 137.4 & 173.4 \\
\hline 7 & 238.1 & 140.8 & 195.5 \\
\hline 8 & 211.1 & 65.4 & 133.5 \\
\hline 9 & 283.9 & 62.7 & 189.9 \\
\hline 10 & 378.1 & 104.0 & 147.8 \\
\hline
\end{tabular}

K-wire: Kirschner wire.

\begin{tabular}{|c|c|c|}
\hline Group & Tensile strength (N) & $p$-value \\
\hline Plate and screws (a) & 246.1 (175.3-452.4) & \multirow[t]{3}{*}{$<0.001(a>c>b)$} \\
\hline K-wire (b) & $134.6(62.7-175.0)$ & \\
\hline $\begin{array}{l}\text { Headless compression } \\
\text { screw (c) }\end{array}$ & $181.2(119.2-211.7)$ & \\
\hline
\end{tabular}

Values are presented as median (range).

K-wire: Kirschner wire.

method, was applied. For the post-hoc test, the Dunn procedure was performed. The level of significance was set at 0.05 and statistical analysis was performed utilizing SAS 9.4 ver. (SAS Inc., Cary, NC, USA).

\section{DISCUSSION}

The treatment goals of metacarpal fractures are the prevention of rotational/angular deformities and shortening and fast and complete recovery of function. ${ }^{13)}$ For these goals to be achieved, precise reduction and internal fixation are important as well as early postoperative joint exercises.
Table 3. Post-hoc Test Based on the Dunn Procedure for Comparison of the Three Operative Methods for Metacarpal Shaft Fractures

\begin{tabular}{lc}
\multicolumn{1}{c}{ Group } & $p$-value \\
\hline Plate and screws vs. K-wire & 0.001 \\
\hline Plate and screws vs. headless compression screw & 0.014 \\
\hline K-wire vs. headless compression screw & 0.022 \\
\hline
\end{tabular}

K-wire: Kirschner wire.

Metacarpal shaft fractures can be treated by closed reduction and casting, but in certain cases incomplete reduction can lead to malunion and failure of synostosis. Also, long-term immobility due to fixation can result in complications such as ankylosis. ${ }^{4,5)}$ Without accurate surgical measurements, the probability of angular deformity, shortening, and rotational deformity increase; in addition, serious aesthetic and functional problems can arise such as cross-fingering and superimposition associated with rotational deformity.

Plate fixation is a commonly used internal fixation technique for a metacarpal shaft fracture. This procedure has the strongest fixation strength ${ }^{14)}$ and allows for early postoperative joint movement. However, it has a high risk for soft tissue damage, fibrosis, and adhesion, which can lead to other complications such as ankylosis, surgery-related infection, and tendon rupture from the plate screws, etc. ${ }^{6,7,10-12)}$ If these complications occur, removing the internal fixation plate and treating the injuries incur a high cost, which becomes an additional burden to the patient.

Percutaneous K-wire fixation is a simple surgical technique that does not require an incision. Therefore, this method has advantages of minimal soft tissue damage and a low infection rate. However, because the fixation strength is weak, the patient may require a splint. Furthermore, restraint on the skin and soft tissue may cause inconvenience during early postoperative joint movement. Also, if the percutaneous $\mathrm{K}$-wire is inappropriately inserted into the tendon, it may interfere with the gliding movement of the tendon and cause ankylosis. ${ }^{4,6}$ Compared to plate fixation, Steinmann pin fixation has less soft tissue damage and a lower risk of extension disability and tendon rupture. However, the strength of fixation is relatively weak, making it difficult to control rotational deformities. ${ }^{15)}$

In order to overcome the disadvantages of plate fixation (soft tissue damage, tendon adhesion, and tendon rupture from the screws) and K-wire fixation (weak fixation), another method was designed using closed 
reduction and percutaneous headless compression screw method. In this cadaveric study, we acquired median values of tensile strength of three operative methods showing significant differences. Also, according to the post-hoc test, we could confirm significant differences in the paired comparisons of the three methods.

The limitations of this study include that although we attempted to use metacarpal bones of similar lengths and widths, there must have been slight differences in length and width, and therefore the same fracture line and angle could not be induced on all bones. In addition, only a total of 30 metacarpal bones were included and divided into ten groups. The small sample size limited statistical analysis. Also, during the measurement of ultimate tensile strength, the bending force was measured, but measurement of rotational movement was not performed.

In this study, we could confirm significant differences between the headless compression screw and K-wire and between the headless compression screw and plate and screws. Even though the fixation strength of headless compression screws was not superior to that of the plate, we think that headless compression screws are sufficiently strong to fix the fracture site according to the results. This study demonstrated that intramedullary headless compression screw fixation can be successfully used in clinical settings with favorable fixation strength in some metacarpal fractures.

From a viewpoint of clinical relevance, we could provide the theoretical grounds for the advantages of headless compression screw fixation in metacarpal shaft fractures including relatively strong fixation, small incision, minimal surgical time, low risk for infection, small soft tissue injuries and early range of motion exercise. Many studies have already attempted to prove clinical usefulness of headless compression screws in metacarpal fractures. Tobert et al. ${ }^{16)}$ suggested that intramedullary headless screw fixation of metacarpal fractures was an efficacious treatment modality for patients with comminution, multiple fractures, malrotation, and those who required rapid mobilization. Ruchelsman et al. ${ }^{17)}$ concluded that limited open retrograde intramedullary headless screw fixation was safe and reliable for metacarpal neck and subcapital and axially stable shaft fractures, allowed for early postoperative motion without affecting union rates, and obviated immobilization. Likewise, several orthopedic clinicians already used headless compression screw fixation for treating metacarpal fractures; however, their methods depended on their experience without any theoretical analysis based a mechanical test.

Despite the advantages, we have to concern about articular cartilage damage of the metacarpal head with use of intramedullary headless compression screw fixation in metacarpal shaft fractures. There may be some problems of this surgical method such as the exposure of the metacarpophalangeal joint, but the $3.0-\mathrm{mm}$ headless compression screws that were used did not protrude out of the articular surface. In addition, minimal articular cartilage damage with use of an appropriate surgical technique could permit early range of motion exercises after surgery. However, small articular cartilage damage could accelerate metacarpophalangeal joint osteoarthritis, and it warrants further research.

In this cadaveric study, we assessed the tensile strength of three fixation techniques in the simulated metacarpal fractures and observed that the median values decreased significantly in the following order: the plate and screw fixation, headless compression screw fixation, and $\mathrm{K}$-wire fixation. Furthermore, significant differences were noted between the headless compression screw fixation and K-wire fixation and between the headless compression screw fixation and plate and screw fixation. Based on such results, we came to the conclusion that the fixation strength of the headless compression screw was stronger than that of the K-wire and weaker than that of the plate and screws. Therefore, we assume that the headless compression screw might be strong enough to fix metacarpal shaft fractures and could be one of the effective surgical treatment methods for metacarpal shaft fractures. To confirm our observation, a clinical study should be conducted.

\section{CONFLICT OF INTEREST}

No potential conflict of interest relevant to this article was reported.

\section{REFERENCES}

1. Reyes FA, Latta LL. Conservative management of difficult phalangeal fractures. Clin Orthop Relat Res. 1987;(214):2330 .

2. Burkhalter WE. Closed treatment of hand fractures. J Hand
Surg Am. 1989;14(2 Pt 2):390-3.

3. Lee YK, Joh BJ, Ahn WI. Percutaneous intramedullary multiple Kirschner-wire fixation for the metacarpal fractures. J Korean Soc Surg Hand. 1999;4(2):278-84. 
Oh et al. Tensile Strength of Three Fixation Methods in Metacarpal Fractures

Clinics in Orthopedic Surgery • Vol. 11, No. 1, 2019• www.ecios.org

4. Kim BS, Cho SD, Cho YS, et al. Operative treatment of metacarpal shaft fracture. J Korean Soc Fract. 1999;12(3):720-6.

5. Jeong CH, Kim HM, Lee KH, Choi MG, Kim YS, Lee DR. Percutaneous intramedullary fixation using prebent Kwires of metacarpal shaft fractures. J Korean Soc Surg Hand. 1999;4(2):199-203.

6. Jones WW. Biomechanics of small bone fixation. Clin Orthop Relat Res. 1987;(214):11-8.

7. Dabezies EJ, Schutte JP. Fixation of metacarpal and phalangeal fractures with miniature plates and screws. J Hand Surg Am. 1986;11(2):283-8.

8. Kozin SH, Thoder JJ, Lieberman G. Operative treatment of metacarpal and phalangeal shaft fractures. J Am Acad Orthop Surg. 2000;8(2):111-21.

9. Melone CP Jr. Rigid fixation of phalangeal and metacarpal fractures. Orthop Clin North Am. 1986;17(3):421-35.

10. Page SM, Stern PJ. Complications and range of motion following plate fixation of metacarpal and phalangeal fractures. J Hand Surg Am. 1998;23(5):827-32.

11. Stern PJ, Wieser MJ, Reilly DG. Complications of plate fixation in the hand skeleton. Clin Orthop Relat Res.
1987;(214):59-65.

12. Fambrough RA, Green DP. Tendon rupture as a complication of screw fixation in fractures in the hand: a case report. J Bone Joint Surg Am. 1979;61(5):781-2.

13. Suh JS, Oh HK. Treatment of metacarpal shaft fractures with plates and screws. J Korean Orthop Assoc. 2003;38(2):199204.

14. Black D, Mann RJ, Constine R, Daniels AU. Comparison of internal fixation techniques in metacarpal fractures. J Hand Surg Am. 1985;10(4):466-72.

15. Day CS. Fractures of the metacarpals and phalanges. In: Wolfe SW, Hotchkiss RN, Pederson WC, Kozin SH, Cohen MS, eds. Green's operative hand surgery. 7th ed. Philadelphia: Elsevier; 2016. 239.

16. Tobert DG, Klausmeyer M, Mudgal CS. Intramedullary fixation of metacarpal fractures using headless compression screws. J Hand Microsurg. 2016;8(3):134-9.

17. Ruchelsman DE, Puri S, Feinberg-Zadek N, Leibman MI, Belsky MR. Clinical outcomes of limited-open retrograde intramedullary headless screw fixation of metacarpal fractures. J Hand Surg Am. 2014;39(12):2390-5. 